\title{
Editorial: Mathematical Modelling of the Pandemic of 2019 Novel Coronavirus (COVID-19): Patterns, Dynamics, Prediction, and Control
}

\begin{abstract}
Hui-Jia $\mathrm{Li}^{1 * t}$, Lin Wang ${ }^{2 \dagger}$, Zhen Wang ${ }^{3 \dagger}$, Zhanwei $\mathrm{Du}^{4 \dagger}$, Chengyi Xia ${ }^{5 \dagger}$, Aristides Moustakas ${ }^{6 \dagger}$ and Sen $\mathrm{Pei}^{7 t}$

${ }^{1}$ School of Science, Beijing University of Posts and Telecommunications, Beijing, China, ${ }^{2}$ Department of Genetics, Faculty of Biology, University of Cambridge, Cambridge, United Kingdom, ${ }^{3}$ Center for OPTical IMagery Analysis and Learning (OPTIMAL), Northwestern Polytechnical University, Xi'an, China, ${ }^{4}$ School of Public Health, Li Ka Shing Faculty of Medicine, The University of Hong Kong, Hong Kong, SAR China, ${ }^{5}$ Tianjin University of Technology, Tianjin, China, ${ }^{6}$ Natural History Museum of Crete, University of Crete, Heraklion, Greece, ${ }^{7}$ Department of Environmental Health Sciences, Mailman School of Public Health, Columbia University, New York City, NY, United States
\end{abstract}

Keywords: COVID-19, epidemic spreading, pattern recognition, mathematical modelling, transmission dynamics, disease prediction

\section{Editorial on the Research Topic}

Editorial: Mathematical Modelling of the Pandemic of 2019 Novel Coronavirus (COVID-19): Patterns, Dynamics, Prediction, and Control

Appeared as a public health event, the COVID-19 pandemic has triggered massive crises in public health systems and economy. The COVID-19 has fleetly spread to most countries of the world. By the end of May 2021, SARS-CoV-2 has infected 180 million people and caused over three million deaths worldwide. To control the outbreak of COVID-19, travel restrictions, economic lockdowns, and border controls have been taken by many countries. The ongoing COVID-19 pandemic motivates the scientific community to contribute to infectious disease modeling, epidemiological study and outbreak prediction. That is, understanding the mechanism of COVID-19 pandemic spreading, exploiting the infection prevention and mitigation, and evaluating policy implementation, are important research questions for academics and policymakers.

To exploit this urgent question, mathematical models have been widely used to analyze the characteristics, impacts and emergency responsefor COVID-19. Mathematical modeling is a kind of mathematical structure that is made up of various concepts and formulas in mathematics, used for generally or approximately expressing the characteristics or quantitative interdependence of a certain system of things.

This special issue has collected a series of studies on epidemic data analysis and supporting decision-making. Those studies fit mathematical models for COVID-19 to analyze the characters of the COVID-19 pandemic in different regions, for instance, Azanza Ricardo et al. investigate the outbreak of COVID-19 in Mexico with different scenarios. While Miao et al. use mathematical modeling to study options for business reopening during the COVID-19 pandemic. As a commonly used epidemiologic model, the Susceptible, Infectious, and/or Recovered (SIR) model, the reproductive number of the SIR model is discussed to assess COVID-19 spread (Silveira et al.; Espinosa et al.; Schlickeiser et al.). Also, a mathematical model is developed to study what role social heterogeneity plays in the formation of complex infection propagation patterns (Maltsev et al.). 
Moreover, statistical analysis has been used to characterize the COVID-19 pandemic and predict the spread trend (Wang et al.; Zhao et al.; Liu et al.). Mathematical models and statistical analysis play important roles in public health emergencies, using these models to analyze and develop strategies for the COVID-19 pandemic is also an essential mission. For example, fast epidemic recognition of the epidemic as soon as it appeared, optimal supply and allocation of medical emergency resources (Wang et al.), and assessing the epidemiological consequences of an emergency evacuation (Butail et al.).

Model estimation also plays an important role in this special issue. Wu et al. propose a generalized-growth model to present the evolution of the number of the total confirmed cases over time. The model provides a simple phenomenological approach, with potential implications for forecasting of the pandemic trend. Zhang et al. quantify the incubation period, transmission rate from close contact to infection, and the properties of multiplegeneration transmission from a detailed database in mainland China. Tiwari et al. sought to provide a prediction of the epidemic peak and to evaluate the impact of lockdown on the epidemic peak shift in India. Peker-Dobie et al. argue that the peak of infected individuals coincides with the inflection point of removed individuals. Recent COVID-19 data and the records for Spanish flu and SARS epidemics confirm this observation. Using multistage models the authors provide an explanation for this time shift. Chae et al. estimate the parameters and the initial infections from fitted values, and quantify the infection rate, the basic reproduction number, and the initial number of infected individuals for a number of countries. Kröger and Schlickeiser argue that the Gauss model is the simplest analytically tractable model that allows us to quantitatively forecast the time evolution of infections and fatalities during a pandemic wave and provide relationships between peak time and width, the transient behavior of doubling times, and reproduction factors. Zhuang et al. built a model to estimate the total number of COVID-19 cases in Wuhan, based on the number of cases detected outside Wuhan city in China, with the assumption that cases exported from Wuhan were less likely underreported in other cities/ regions. Total cases are determined by the maximum log likelihood estimation and Akaike Information Criterion (AIC) weight.
This special issue aims to understand the impact of COVID-19 spreading on public health, society, and economics, and provides efficient reference values for economic redevelopment and social stability. So far, the authors have fitted mathematical models for COVID-19, studied the connectedness of the global COVID-19 network across countries, captured the virus transmission among cities, analyzed the dynamic characteristics of the COVID-19 pandemic, assessed the impact of COVID-19 spreading, and predicted the spread trends of COVID-19, which alleviates the public panic caused by COVID-19, grasps the trend of COVID19 transmission, and assists the government or policymakers to make efficient decisions according to the spreading situation of the COVID-19 pandemic.

\section{AUTHOR CONTRIBUTIONS}

All authors listed have made a substantial, direct, and intellectual contribution to the work and approved it for publication.

\section{FUNDING}

This work is supported by Fundamental Research Funds for the Central Universities of China (No. 2020XD-A01-2), NSFC (No. 71871233).

Conflict of Interest: The authors declare that the research was conducted in the absence of any commercial or financial relationships that could be construed as a potential conflict of interest.

Publisher's Note: All claims expressed in this article are solely those of the authors and do not necessarily represent those of their affiliated organizations, or those of the publisher, the editors and the reviewers. Any product that may be evaluated in this article, or claim that may be made by its manufacturer, is not guaranteed or endorsed by the publisher.

Copyright $\odot 2021$ Li, Wang, Wang, Du, Xia, Moustakas and Pei. This is an openaccess article distributed under the terms of the Creative Commons Attribution License (CC BY). The use, distribution or reproduction in other forums is permitted, provided the original author(s) and the copyright owner(s) are credited and that the original publication in this journal is cited, in accordance with accepted academic practice. No use, distribution or reproduction is permitted which does not comply with these terms. 